\title{
Atherosclerotic plaque characterization using plaque area variation in IVUS images during compression: a computational investigation
}

\author{
Matheus Cardoso Moraes*, Fernando Mitsuyama Cardoso, Sérgio Shiguemi Furuie
}

\begin{abstract}
Introduction: The rupture of atherosclerotic plaques causes millions of death yearly. It is known that the kind of predominant tissue is associated with its dangerousness. In addition, the mechanical properties of plaques have been proved to be a good parameter to characterize the type of tissue, important information for therapeutic decisions. Methods: Therefore, we present an alternative and simple way to discriminate tissues. The procedure relies on computing an index, the ratio of the plaque area variation of a suspecting plaque, using images acquired with vessel and plaques, pre and post-deformation, under different intraluminal pressure. Numerical phantoms of coronary cross-sections with different morphological aspects, and simulated with a range of properties, were used for evaluation. Results: The outcomes provided by this index and a widely used one were compared, so as to measure their correspondence. As a result, correlations up to $99 \%$, a strong agreement with Bland-Altman and very similar histograms between the two indices, have shown a good level of equivalence between the methods. Conclusion: The results demonstrated that the proposed index discriminates highly lipidic from fibro-lipidic and calcified tissues in many situations, as good as the widely used index, yet the proposed method is much simpler to be computed.
\end{abstract}

Keywords Intravascular Ultrasound (IVUS), Atherosclerosis, Intravascular elastography, Phantoms, Strain map.

\section{Introduction}

Atherosclerotic plaque rupture is the main reason of acute coronary events. In United States of America (USA) 785,000 new coronary attacks occurs annually, with 470,000 recurrences. A coronary event happens every 25 seconds with one death per minute. Moreover, in 2007 and 2008 the total cost of cardiovascular disease and stroke of USA were U\$286 and U\$297.7 billions, respectively, greater than costs of other diseases (Roger et al., 2012; Rosamond et al., 2007).

Atherosclerosis is the accumulation of lipidic, fibrous, and calcified tissues in the arterial wall. An atherosclerotic plaque rupture may lead to myocardial infarction, unstable angina, or sudden cardiac death (Davies, 2000; Falk et al., 1995; Maurice et al., 2005; Viermani et al., 2000). The lesion severity is directly related to plaque composition and morphological features. A subset of vulnerable plaque is recognized by having large lipid pool with macrophages, covered by a thin cap (Baldewsing et al., 2004; Davies, 1996; Viermani et al., 2000). Accordingly, by identifying the preponderant tissue in a suspecting plaque, very important lesion information is revealed, as the kind of tissue is related to the dangerousness. Consequently, this information provides extra aspects for more accurate therapeutic decision or interventional

*e-mail: matheuscardosomg@hotmail.com

Received: 6 August 2013 / Accepted: 4 December 2013 procedure (Fisher et al., 2000; Ohayon et al., 2001). Consequently, tools and methods dedicated to intravascular ultrasound (IVUS) have been growing rapidly (De Korte and Van der Steen, 2002; Le Floc'h et al., 2009; Maurice et al., 2005).

IVUS is a popular imaging modality for cardiac interventional procedures. Its images supply the cardiologists with anatomical, morphological and pathological coronary and plaque information, crucial for diagnostics, evaluations, and treatment planning (Baldewsing et al., 2004; Liang et al., 2009). Nonetheless, to accurately infer about atherosclerotic plaque composition and dangerousness, additional procedures, such as segmentation and intravascular Elastography, are important (Baldewsing et al., 2004; Cardenas et al., 2013; De Korte and Van der Steen, 2002; Le Floc'h et al., 2009; Maurice et al., 2005; Moraes and Furuie, 2010, 2011).

Intravascular Elastography is a method in which the mechanical properties of tissues from the arterial wall are assessed through strain or elasticity (i.e. shear or Young's modulus) map (Céspedes et al., 1993; Loree et al., 1994; Maurice et al., 2005; Ophir et al., 1991). Therefore, extra information about tissue composition and vulnerability is provided, as a result 
the best therapeutic procedure can be carried out (Céspedes et al., 1993; Ophir et al., 1991). Basically, the Elastography procedure is divided into three main blocks: First, the images are acquired with a variation of intraluminal pressure - usually arterial pressure and inflated balloon are used as source of pressure (De Korte et al., 1999; Shapo et al., 1996). Second, the displacements of microstructures are computed, followed by the strain map computation. Finally, by knowing the intraluminal pressure and resultant strain map, Finite Element Method (FEM) is usually applied to calculate the corresponding force vector and consequent elasticity map, the Young's Modulus of structures (Baldewsing et al., 2004; De Korte and Van der Steen, 2002; Fisher et al., 2000; Le Floc'h et al., 2009; Ohayon et al., 2001).

Prior approaches have dedicated efforts to build well-documented and widely recognized materials in coronary and plaque functional behavior (Baldewsing et al., 2004; Céspedes et al., 1993; De Korte and Van der Steen, 2002; Fisher et al., 2000; Le Floc'h et al., 2009; Loree et al., 1994, Maurice et al., 2005; Ohayon et al., 2001; Ophir et al., 1991). As a result, anatomical and mechanical aspects such as Cap thickness, lesion disposition and parameter values are known. Hence, new tools and methods in Elastography have been created, providing alternative tools for therapeutic decisions, evaluation, and interventional procedures (Fisher et al., 2000; Ohayon et al., 2001). Although precise and reliable, IVUS Elastography is still a costly and complex method to be implemented. Therefore, simple and reliable alternatives for extracting mechanical properties of tissue are needed.

The proposed approach presents a simple and more practical procedure for extracting mechanical properties of suspecting atherosclerotic tissues. The method can be an alternative choice to IVUS Elastography. In our procedure the mechanical properties and corresponding composition of tissues of a suspecting lesion, can be estimated by computing the area ratio of the plaque under inspection in IVUS images (Figure 1). Therefore, during imaging acquisition (Figures 1a and b), different pressure is applied by an expandable balloon to acquire the deformed and non-deformed images. Thus, a distinguishable deformation ratio is assured, and problems related to uncertainty of intraluminal pressure, catheter eccentricity and inclination are well overcome. After, the Plaque Area Computation (Figures 1c and d), the plaque areas under inspection in the IVUS images are segmented and the area of a suspecting plaque quantified in different pressures. Next, Ratio of the Plaque Area Variation, the overall percentage of plaque deformation is calculated (Cardoso et al., 2012). As a result, the type of the predominant tissue of the suspecting area is estimated based on the regional deformation values. Therefore, the goal of this paper is to introduce the procedure, and using deformable numerical phantoms, demonstrating the capability of tissue discrimination of the proposed index, Plaque Area Variation $(A R)$, in a range of situations.

\section{Methods}

The materials used for this work are comprised by a personal computer with an Intel Core 2 Duo, and microprocessor of $2.53 \mathrm{GHz}, 4 \mathrm{~GB}$ of RAM, Windows 764 bits, MATLAB® (2009a) (MathWorks, Inc., Natick, MA, USA) with Imaging Processing and Partial Differential Equation Toolbox. Due to computational models advancements (Hoskins, 2008), numerical phantoms were used. The phantoms, deformation outcomes and gold standard strain maps were obtained by the framework described by Cardoso et al. (2012). The complete method is embedded in the Toolbox called IVUSSim. It is a citationware software, available online at $h t t p: / / w w w$. leb.usp.br/IVUSSim, free of charge for research and educational purposes (Cardoso et al., 2012). A brief explanation of the framework is given below.

\section{Overview of IVUSSim}

Realistic IVUS image generation in Different Intraluminal Pressures (IVUSSim) - The framework is a tool, in which from different anatomical and morphological coronary cross-section models and input parameters, IVUS phantoms are created (Figure 2e). In addition, some related results, such as the corresponding non-deformed and deformed gray level images (Figure 2c), strain maps (Figure 2d) are also generated to serve as gold standard to many investigations (Cardoso et al., 2012). In summary, the framework works as follows: First the user selects an available, or designs a new coronary cross section model, representing a coronary cross section in a diastole cardiac phase (Figure 2a). Second, the investigator sets some parameters, such as ultrasound frequency, number of transducers, and incremental intraluminal pressures. Third, the algorithm automatically identifies the different regions of the selected model, such as media, adventitia, and plaques, and asks the user to insert the corresponding mechanical properties, such as Young's Modulus, for each region. Next, a 2D mesh is constructed, and FEM is carried out, so that the deformed mesh is generated (Figure 2b). Finally, a morphism procedure is performed; hence, infinitesimal or very small structures, such as scatterers and pixels, are repositioned according to FEM deformation. As a result, the correspondent mesh and deformed mesh 


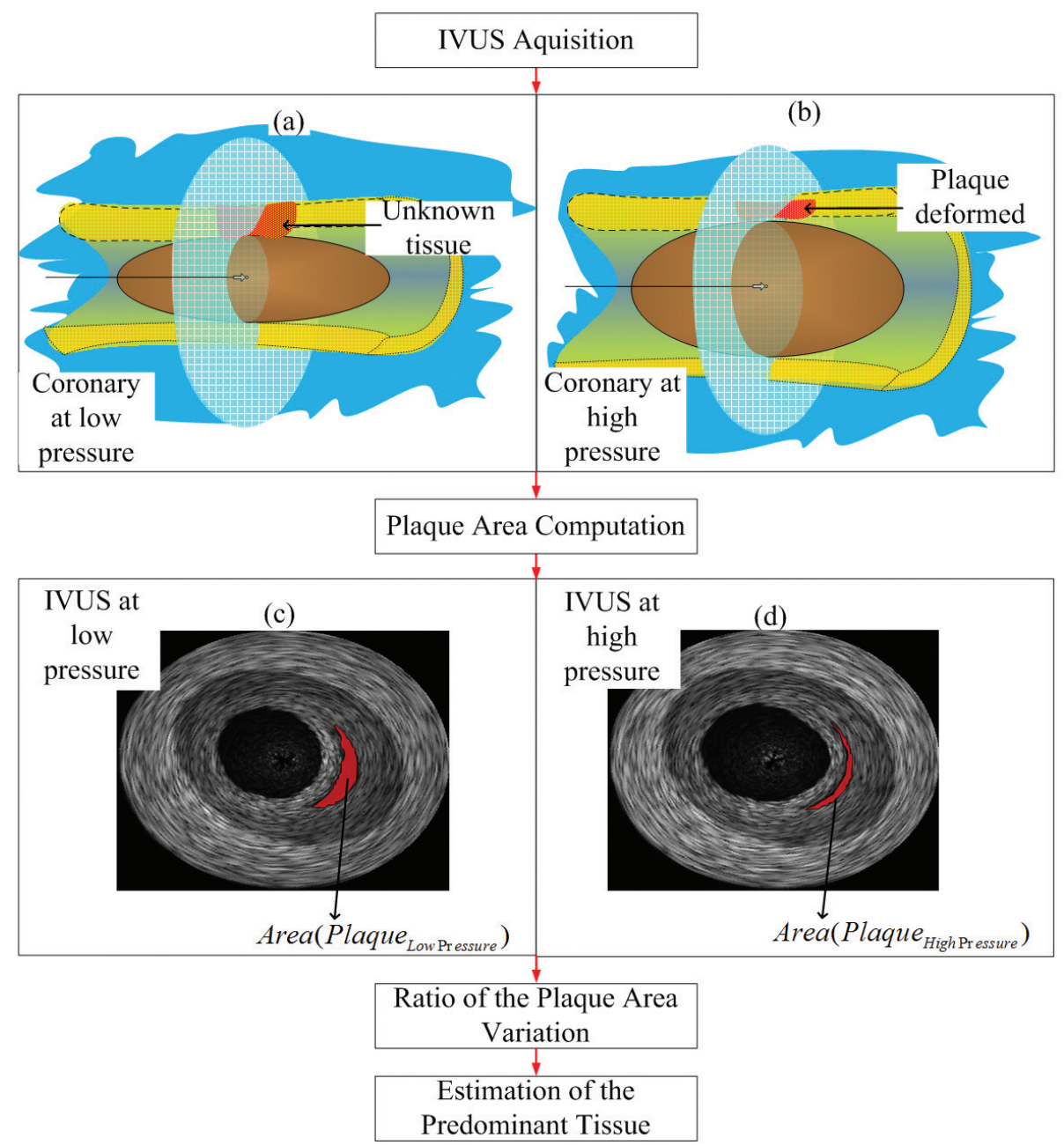

Figure 1. Block diagram of the proposed procedure. An illustration of an IVUS acquisition with a compliant balloon in: (a) low pressure; (b) high pressure. IVUS phantoms corresponding to the previous acquisition at: (c) low pressure; (d) high pressure.

led to the gray level images and realistic phantoms, respectively (Figures $2 \mathrm{c}$ and e). In addition, as the displacement of small structure is known, the strain map is also generated (Figure 2d). Therefore, the gray level images, and strain map (Figures $2 \mathrm{c}$ and d) can be used as gold standards for investigation of structural deformations and shifting of small particles, such as speckle or pixel. For more information about the phantom creation framework, please refer to Cardoso et al. (2012).

\section{Deformation measurements}

Two different methods are used to compute the level of plaque deformation, the Average of the Plaque Radial Strain Values $(A S)$ (Figure 2d), and the proposed method, the Ratio of the Plaque Area Variation $(A R)$ (Figure 2c). The $A R$ outcomes are obtained by:

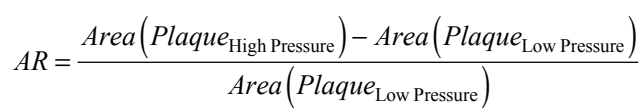

where, Area (Plaque $\left.e_{\text {Low Pressure }}\right)$ and Area $\left(\right.$ Plaque $\left._{\text {High Pressure }}\right)$ are the plaque areas with low, and high intraluminal pressure, respectively. The plaque areas were obtained from the gray level images (Figure 2c), since they are the images representation of the mesh and deformed mesh, gold standards, the plaque under investigation in low and high pressure are isolated and their area computed. The $A S$ results are extracted from the corresponding radial strain map by:

$A S=\bar{\varepsilon}_{\text {plaque }} \pm \sigma_{\text {plaque }}$

where, $\bar{\varepsilon}_{\text {plaque }}$, and $\sigma_{\text {plaque }}$ are the mean and standard deviation of the plaque radial strain map values, respectively (Figure 2d). 


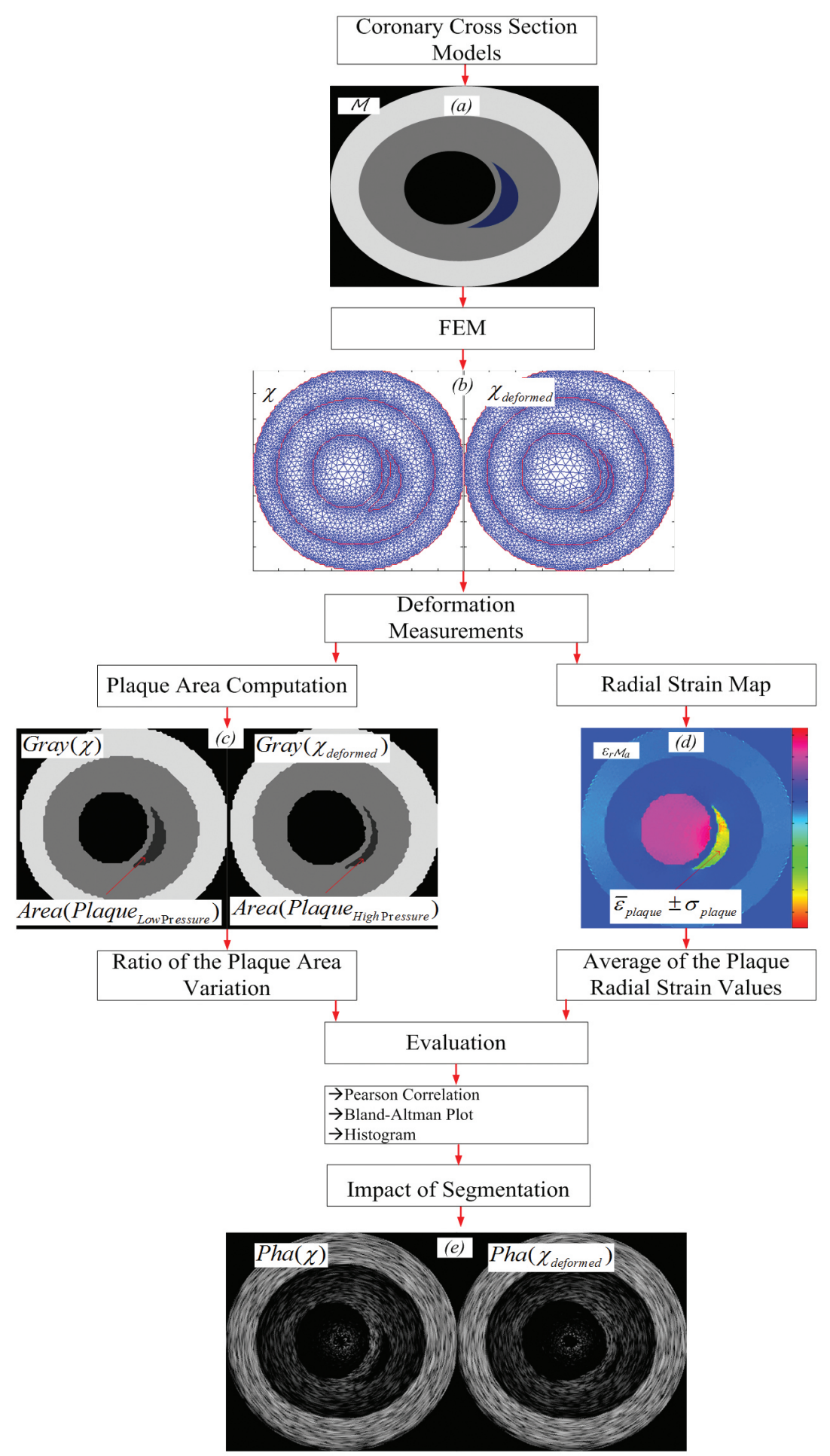

Figure 2. Block diagram of the overall methodology of this approach: (a) A coronary cross section model under evaluation (M); (b) The mesh, $\chi$, and deformed mesh, $\chi_{\text {deformed }}$, of M, after FEM; (c) The corresponding gray level images of the mesh, $\chi$, and deformed mesh, $\chi_{\text {deformed }}$, $\operatorname{Gray}(\chi)$ and Gray $\left(\chi_{\text {deformed }}\right)$, respectively; (d) Strain Map corresponding to the resultant deformation; (e) The resultant non-deformed and deformed IVUS phantoms. 


\section{The investigation}

Using IVUSSim, the present approach aims to demonstrate computationally the potential of the proposed index, $A R$, for atherosclerotic tissue characterization. In addition, the index reliability is reinforced, by comparing its results with the corresponding outcomes obtained by strain map computations, a widely used index, which can be found in many studies (Baldewsing et al., 2004; Le Floc'h et al., 2009; Maurice et al., 2005; Shapo et al., 1996). Specifically, the phantoms were generated with ultrasound frequency at $20 \mathrm{MHz}$ and transducer with 256 RF-lines and the tissue acoustic parameters of Table 1. The FEM was carried out in a set of coronary cross section models under a variation of conditions, such as different cap-thickness values, and Young's modulus. The deformation results of each kind of plaque, highly-lipidic, fibro-lipidic, and calcified, were computed by the two indexes, so that they could be directly compared. The overall procedure of this approach is summarized as follows (Figure 2). First, a Coronary Cross Section Model (Figure 2a) is chosen from a set of models to be investigated (Figure 3). Second, FEM was performed to simulate the different physiological coronary and plaque behaviors (Cardoso et al., 2012). Third, the two deformation measurements, $A R$ (Figure 2c) and $A S$ (Figure 2d) were used to obtain the plaque deformation values. Finally, the correspondence between the two methods and the reliability of the proposed method were directly related, and statistically corroborated during the Evaluation (Figure 2). The evaluation was performed by computing and comparing the deformation values obtained by the proposed method, $A R$, and a well known method, strain map computations. Pearson correlation, Bland-Altman plot, and the Histogram of the deformation values computed by the two indices were carried out so that the reliability of the proposed method could be analyzed. Finally, the results obtained by the gold standards, the gray level images, were compared to the corresponding outcome obtained by the segmented phantoms; thus, the impact of segmentation for highly-lipidic plaque could be measured.

\section{Coronary cross section models}

A set of coronary cross section models, with a variation of morphological features, was created to have the coronary plaque deformities investigated (Figure 3 ). The set of models represents the anatomical shape of artery coronary cross sections in a diastole cardiac phase. The adventitia is represented by the lighter gray region, the media is the dark gray, and the blue, brown and white represent the highly-lipidic, fibro-lipidic, and calcified plaques, respectively (Figure 3). Each model was simulated in different morphological situations. The morphological situations correspond to the different kinds of plaques and their disposition in the coronary with the cap thickness values, $C a p=100,200$, and $300 \mu \mathrm{m}$, common values employed by apposite studies (Baldewsing et al., 2004; Le Floc'h et al., 2009; Maurice et al., 2005). Therefore, the plaque behavior when the plaque is isolated, and when it is neighboring another plaque, with different cap thickness, could be investigated. Specifically for models $M_{f}$ and $M_{i}$ (Figures $3 \mathrm{f}$ and i), the calcified plaque is in front of the highly-lipidic and fibro-lipidic plaques, respectively. In a real IVUS image, the calcified tissue would produce a shadow covering the others plaques. For this reason, the analysis of the highly-lipidic and fibro-lipidic plaques in this situation would be unfeasible, since investigators and methods wouldn't be able to see them. However, the goal here, by using these two models, is to investigate the calcified plaque behavior with other neighbor's tissues. Since the calcified can be identified in real IVUS images and in the phantoms, the investigation could be performed.

\section{Coronary and plaques parameters for performing FEM}

The physiological coronary and plaque properties and behaviors were numerically represented. In order to do that, the coronary and plaque parameters used in

Table 1. Acoustic and mechanical tissue parameters used in the Models.

\begin{tabular}{lccc}
\hline & \multicolumn{3}{c}{ Parameters } \\
\cline { 2 - 4 } Tissue & \multicolumn{2}{c}{ Acustic } & Mechanical \\
\cline { 2 - 4 } & Attenuation [dB/(cm.MHz)] & Acoustic impedance [MRayl] & Young's modulus [kPa] \\
\hline Adventicia & 1.57 & 1.81 & $80 \mathrm{kPa}, 150 \mathrm{kPa}, 300 \mathrm{kPa}, 450 \mathrm{kPa}, 600 \mathrm{kPa}$ \\
Media & 1.09 & 1.62 & $600 \mathrm{kPa}$ \\
Higly lipidic & 0.48 & 1.40 & $25 \mathrm{kPa}$ \\
Fibro lipidic & 0.54 & 1.63 & $296 \mathrm{kPa}$ \\
Calcified & 6.90 & 7.38 & $1500 \mathrm{kPa}$ \\
\hline
\end{tabular}




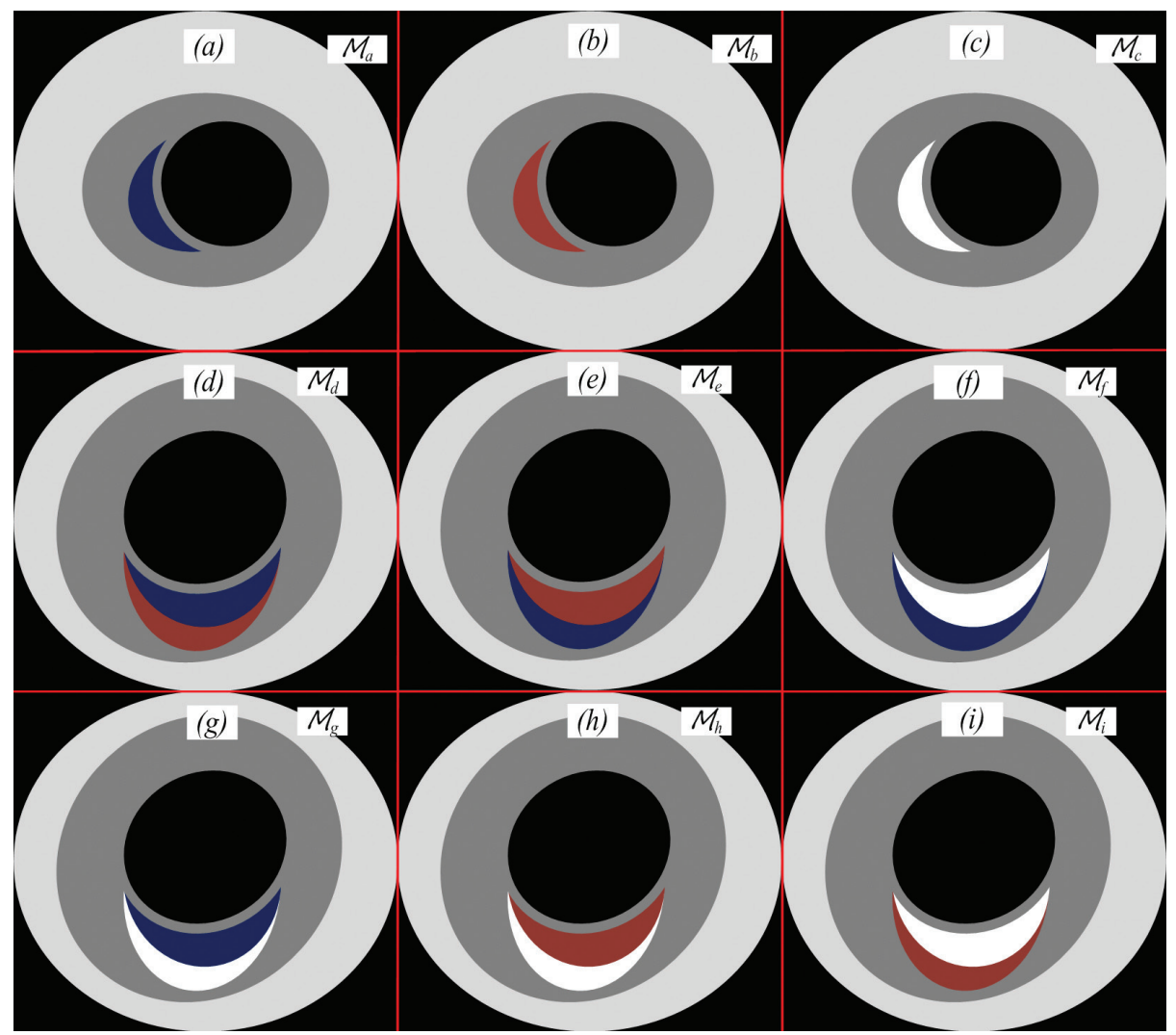

Figure 3. Coronary cross section models (Mn) to investigate: (a) The highly-lipidic plaque; (b) The fibro-lipidic plaque; (c) The calcified plaque; (d) Highly-lipidic with a fibro-lipidic plaque neighbor; (e) The fibro-lipidic with a highly lipidic plaque neighbor; (f) The calcified with a highly-lipidic neighbor; (g) The highly-lipidic with a calcified plaque neighbor; (h) The fibro-lipidic tissue with a calcified plaque neighbor; (i) The calcified with a fibro-lipidic neighbor.

IVUSSim, were extracted by a number of previous investigations (Baldewsing et al., 2004; Cardoso et al., 2012; Le Floc'h et al., 2009; Zienkiewicz et al., 2010a, 2010b). The mesh was generated with triangular element, using Delaunay triangulation. To maximize precision, a higher mesh density was disposed at the borders (Figure 2b). The intraluminal forces were applied perpendicularly to the lumen border, corresponding to a compliant balloon producing from 1 to $0.05 \mathrm{~atm}$ of pressures. The minimum 0.05 corresponds approximately to $40 \mathrm{mmHg}$, which is the normal difference between systolic and diastolic cardiac pressure. The maximum of $1 \mathrm{~atm}$ was chosen, because level of pressure provides a good tradeoff between high deformation ratio and risk of plaque rupture during acquisition procedures. Moreover, by using the balloon, problems with catheter eccentricity and inclination are overcome, ensuring the reliability of the deformed values (Choi et al., 2002; De Korte et al., 1999; Shapo et al., 1996). The fixed nodes, at the external border, after the adventitia tissue, were selected to be the boundary condition. The arterial and plaque mechanical properties used were: Poisson's ratio, $v=0.49$, and Young's modulus, $\mathrm{E}=600 \mathrm{kPa}, 1500$ $\mathrm{kPa}, 296 \mathrm{kPa}$, and $25 \mathrm{kPa}$, for the media, calcified, fibro-lipidic, and highly-lipidic with macrophages, respectively. As the adventitia may have a variation of elasticity, 5 values were considered for simulation $E_{\text {adventitia }}=80,150,300,450,600 \mathrm{kPa}$. All parameters values are correspondent to mechanical properties of in vivo coronaries, and were obtained from previous related studies (Table 1) (Baldewsing et al., 2004; Cardoso et al., 2012; De Korte and Van der Steen, 2002; Le Floc'h et al., 2009; Shapo et al., 1996; Zienkiewicz et al., 2010a, 2010b).

\section{Results}

We evaluated the reliability of the proposed index by analyzing two aspects, the correlation between, $A R$ and $A S$, as well as the equivalence between deformation ratios and corresponding tissues. The deformation values of $A R$ and $A S$ for the three plaques using nine models (Figure 3 ) in different situations 
were acquired at 6 different pressures, $1,0.75,0.5$, $0.25,0.1,0.05 \mathrm{~atm}$. The Models, $M_{\mathrm{a}}, M_{\mathrm{d}}$, and $M_{\mathrm{g}}$ (Figures 3a, d, and g), were devoted to deformation values of the highly-lipidic plaque, whereas $M_{\mathrm{b}}, M_{\mathrm{e}}$, $M_{\mathrm{h}}$, and $M_{\mathrm{c}}, M_{\mathrm{f}}, M_{\mathrm{i}}$ were dedicated to the fibro-lipidic, and calcified plaque ones, respectively (Figures $3 \mathrm{~b}$, $\mathrm{e}, \mathrm{h}$, and c, f, i). In addition, different morphological situations were also considered for computing the deformation outcomes, for instance, Cap $=100$, $200,300 \mu \mathrm{m}$, and adventitia elasticity $\mathrm{E}_{\text {adventitia }}=80$, $150,300,450,600 \mathrm{kPa}$. The entire simulation for the different models and morphological situations led to 135 values for each pressure, for instance Table 2 shows the values for $1 \mathrm{~atm}$ of pressure, which were computed by Equations 1 and 2. As it can be observed in Table 2, the greatest majority of $A R$ values are very close and inside the mean and standard deviation of the $A S$ index, showing a strong correspondence between them.

\section{Comparison}

The evaluation was performed by: First computing the Pearson Correlation between each $A R$ result with the corresponding $A S$ (reference method); Second, analyzing residuals using Bland Altman plot; Third verifying the separability among classes (Histogram) for the deformation outcomes of each of the 6 applied pressures (Figures 4, 5 and 6). In addition, the impact of segmentation, for highly-lipidic plaque classification, was measured.

Pearson Correlation is an index which measures how similar two sets of data are; the level of correlation is denoted by $(\rho)$, and the closer it is to 1 , the higher is the correlation. Specifically, the Pearson Correlation was computed, and the correspondences between $A R$ and $A S$, for the results of the 6 pressures were obtained (Figures 4). As can be observed in Figure 4, there are two predominant clusters, as highlighted in Figure 4a: bottom-left - cluster of highly-lipidic values; top-right: the other tissues, for instance fibrolipidic and calcified. The two clusters can be noticed for the six applied pressures. The linear aspect of the clusters in all cases (Figure 4) demonstrates the strong correspondence between the two indexes, $A R$ and $A S$. It proved the strong relationship between the $A R$ and $A S$ values, for highly-lipidic, fibro-lipidic calcified tissue results. In addition, a correlation, $\rho$, very close to 1 in practically all cases (Figure 4 ), with a linear correlation $(A R \approx 1.2 A S)$, strongly corroborate the two indexes proportionality.

The Bland Altman plot provides information about the level of agreement between two sets of data, which are devoted to measure a common property; the more points inside the limits, the greater the agreement between two indices. In our evaluation, the
Bland Altman plot was performed between $A R$ and $A S$ also for the 6 pressures (Figures 5). As expected, it could be identified two major clusters of data, the data provided by the highly-lipidic and other tissues deformations, highlighted in Figure 5a. As can be seen in Figure 5, the plots, for all pressures, show the immense majority of points inside the limit of agreement, indicating the strong agreement between $A S$ and $A R$ outcomes.

The Histogram is a representation of data distribution. By carrying out the histogram of a dataset in different situations, besides the frequency of occurrence, we can visually demonstrate the tendency in shape and direction of each cluster. Additionally, it permits the computation of some related parameters, such as separability $(\eta)$, and the difference in distance between clusters $(\Delta(\%))$. Again, in this evaluation, the Histogram for $A R$ and $A S$ data, for the 6 applied pressures was computed (Figure 6). As can be observed in Figure 6, the highly-lipidic and other tissue clusters of deformation values can be well identified in both, the $A R$ and $A S$ histogram, and for all applied pressure. In addition, both indexes, $A R$ and $A S$, provide similar distributions (Figure 6). Therefore, the high correlation between the proposed and usual method are reinforced.

\section{Highly-lipidic discrimination}

It can be assumed that the $A R$ distributions discriminate better the tissues than the values computed by $A S$ (Figure 6). As can be observed in Figure 6, the higher average of highly-lipidic distribution of $A R$ led to a greater difference between the highly-lipidic and the other cluster. We also analyzed the discrimination capability of $A R$ by computing two indices, the histogram class separability, $\eta$, (Otsu, 1979), and the difference in distance between classes of tissue $(\Delta(\%))$. The $\eta$ quantifies how two clusters of data can be well discriminated; it varies from 0 to 1 , the greater the separability, the better the method is for classes discrimination. The $\Delta(\%)$ provides the minimum distance between edges of two classes, illustrated in Figures $6 \mathrm{a}$ and $\mathrm{b}$. Both indices, $\eta$ and $\Delta(\%)$ were computed between the two clusters for the two indices, $A R$ and $A S$, and for all pressures (Figure 6). As can be observed in Figure 6, $\eta$ is almost the same, while $\Delta(\%)$ for the $A R$ values is higher than for the $A S$ in all cases. Consequently, highly-lipidic plaque can be reliably identified by the proposed method.

\section{Impact of segmentation for highly-lipidic discrimination}

Phantoms from the models with lipidic plaques were created for every pressure, using the parameters described in Table 1. The highly-lipidic plaques 


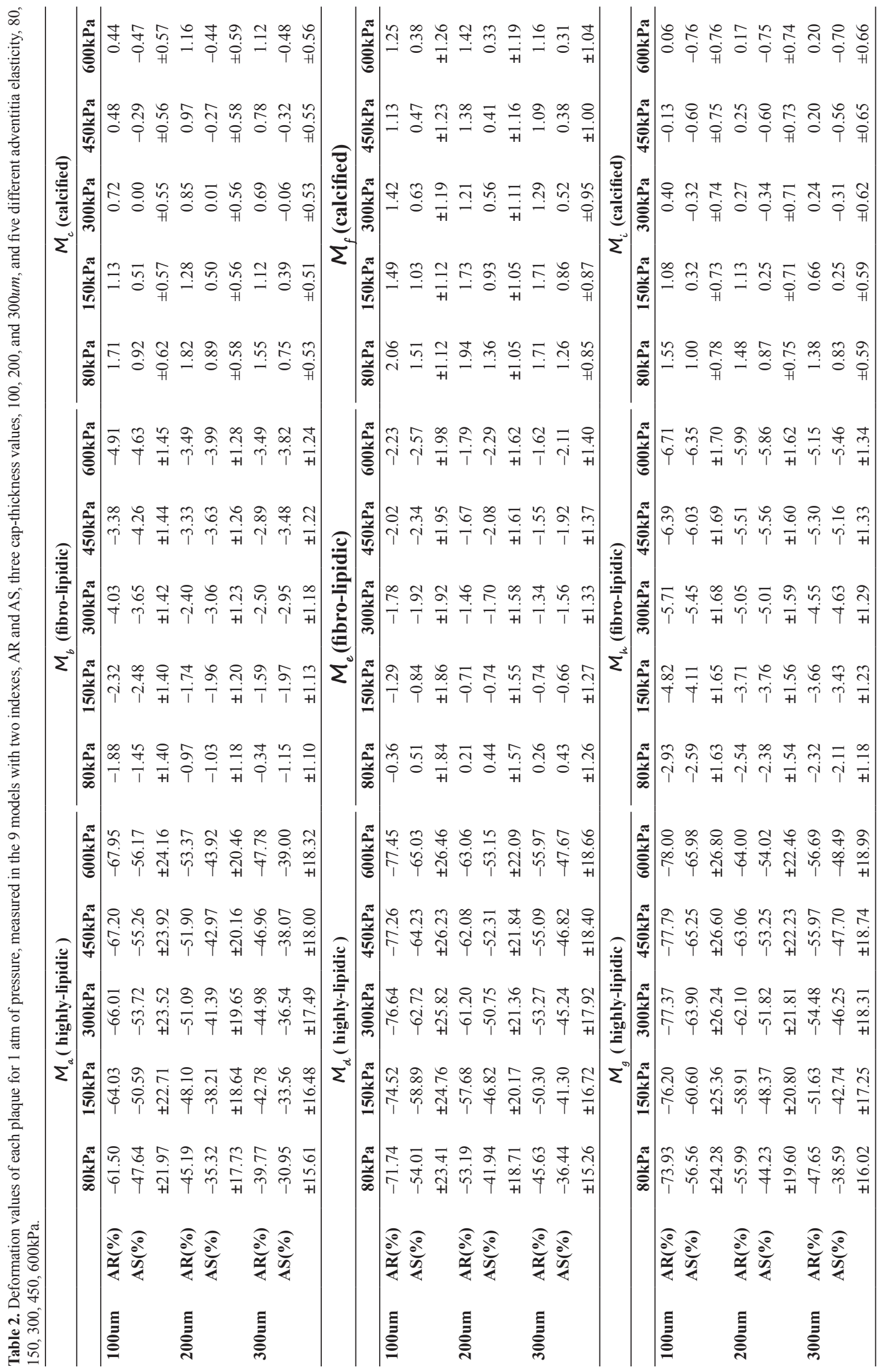



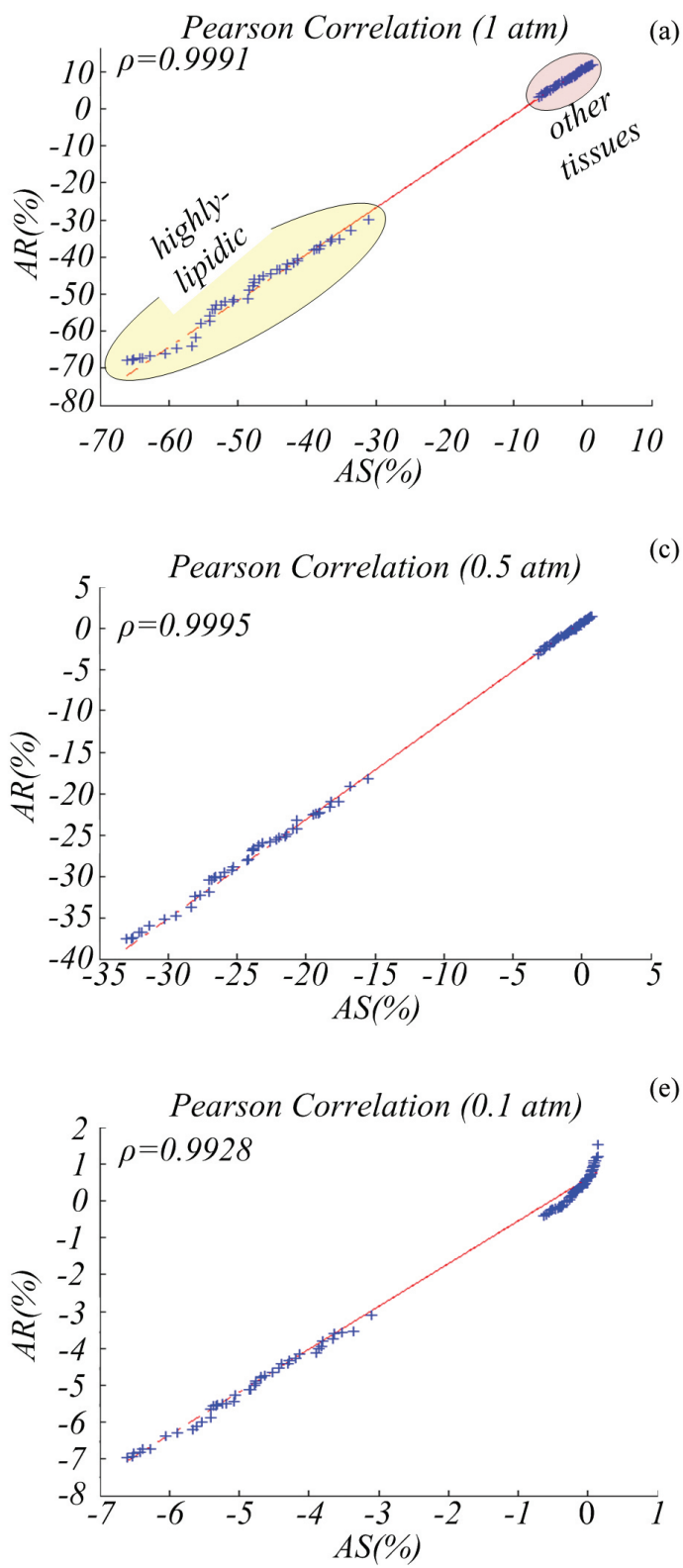

(a)

(c)

e)
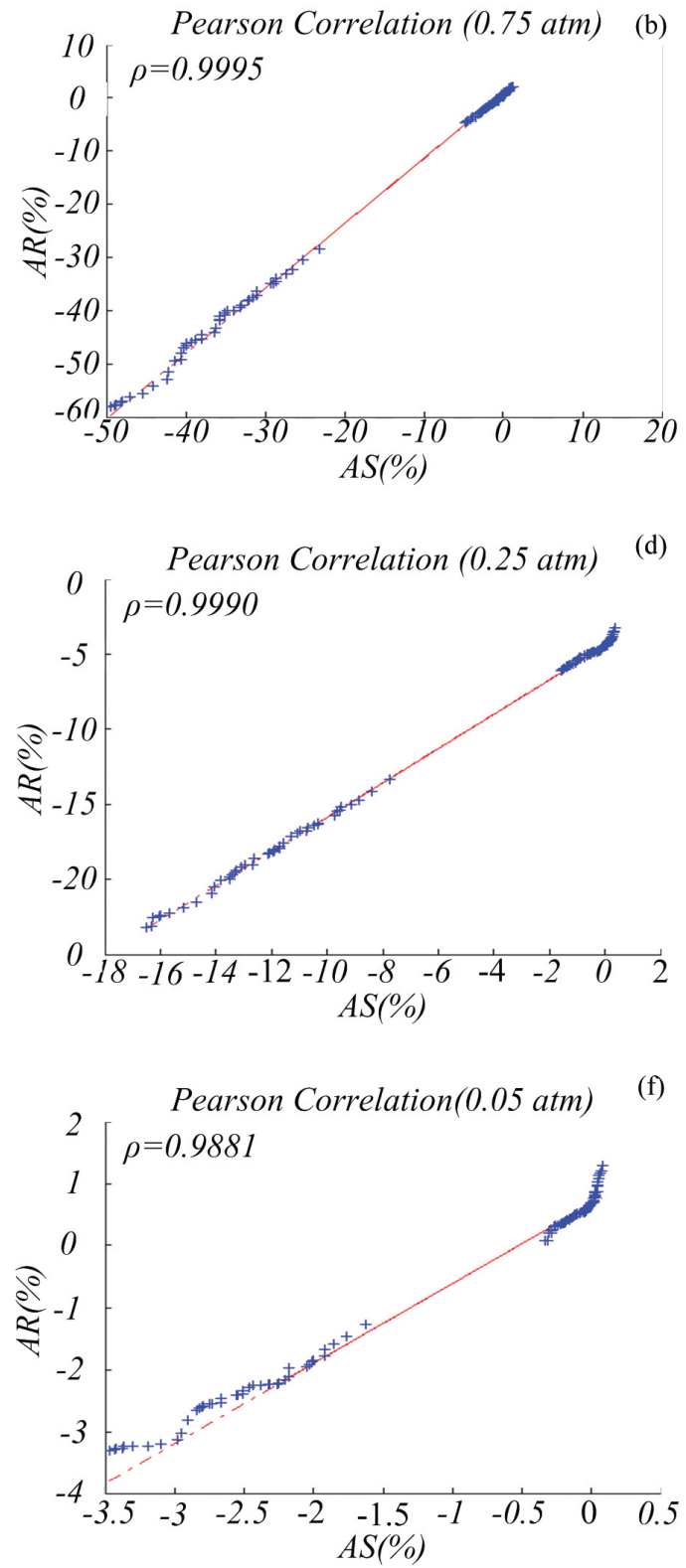

Figure 4. Pearson Correlation between AR and AS values for pressures of: (a) $1 \mathrm{~atm}$; (b) $0.75 \mathrm{~atm}$; (c) $0.5 \mathrm{~atm}$; (d) $0.25 \mathrm{~atm}$; (e) $0.1 \mathrm{~atm}$; (f) $0.05 \mathrm{~atm}$.

were manually segmented by specialists (Figure 7b). Once the phantoms were ready two investigators, with knowledge in IVUS image features and segmentation, segmented manually in agreement to each other, the corresponding lipidic regions of the phantoms. Once the corresponding areas were obtained, the Ratios of the Plaque Area Variation were computed for the segmented plaques. The impact of segmentation (IS) was obtained by computing the error, using:

$I S=\left|A R-A R_{\text {Segmented }}\right|$ where, $A R$ and $A R_{\text {Segmented }}$, are the area ratios computed by the gold standards (Figure 7a), and corresponding segmented images (Figure 7b), respectively, and $I S$ is the impact or error of segmentation. The obtained IS result, was $4.3 \pm 3.3 \%$. This value may only influence the discrimination between highly-lipidic and other tissues, for pressures equal and below $0.25 \mathrm{~atm}$, where the pressure does not cause enough deformation to overcome the discrimination between highly lipidic and other tissues. However, better segmentation result can be obtained using other segmentation approaches, or with a good preprocessing. 


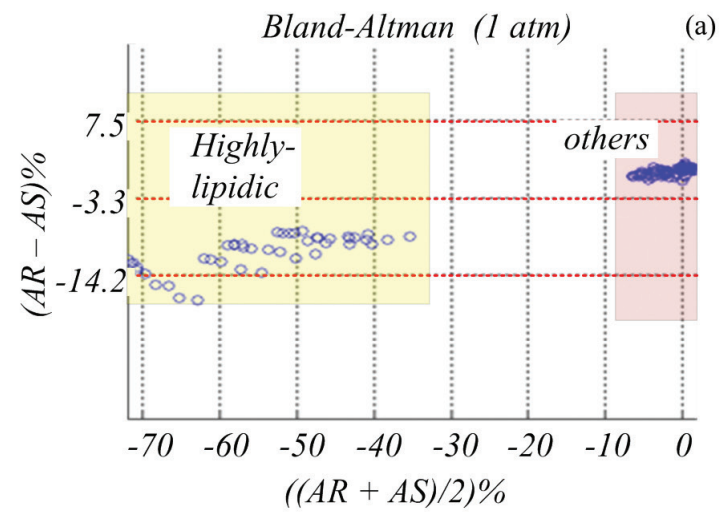

\section{(a)}
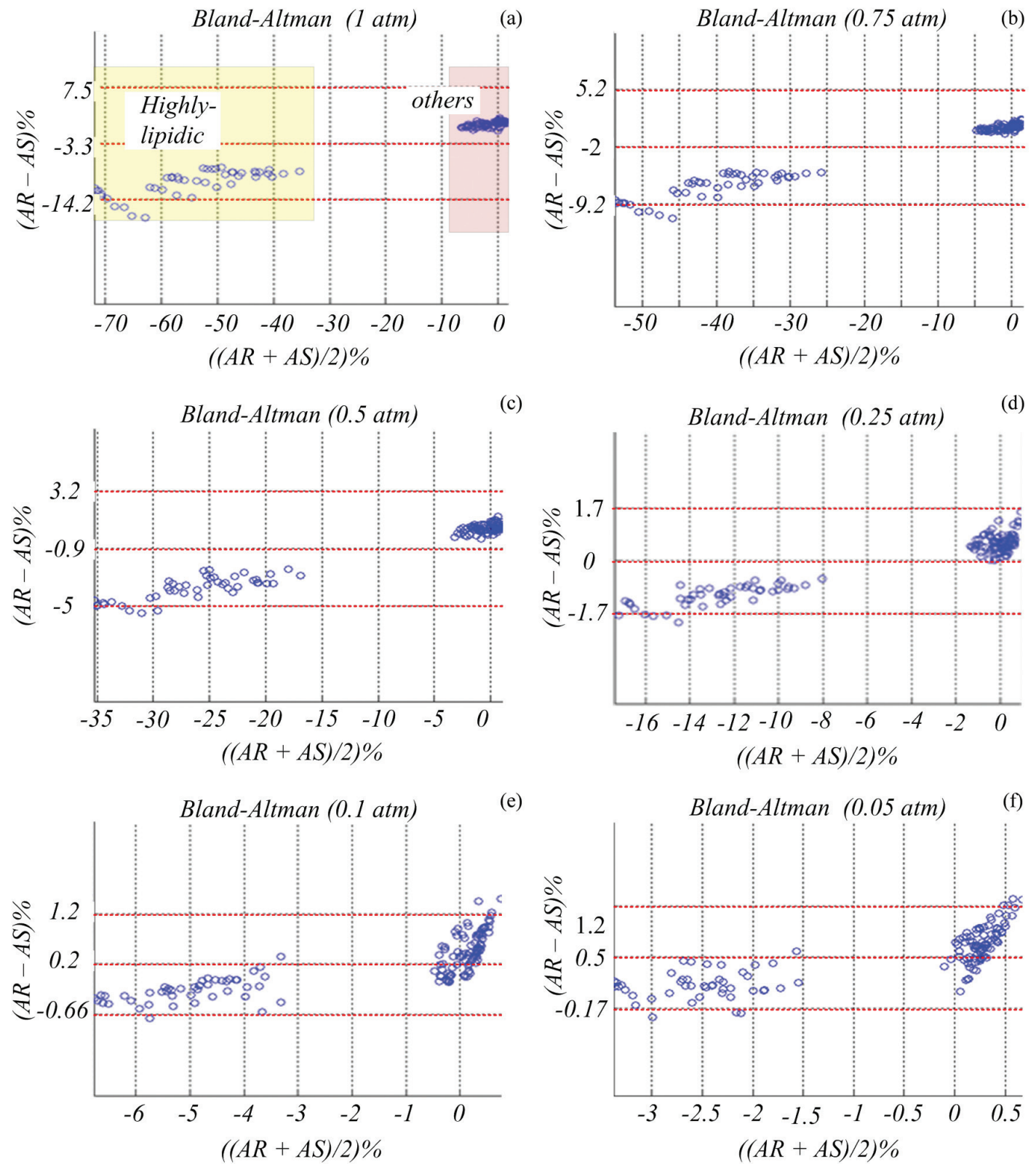

(e)

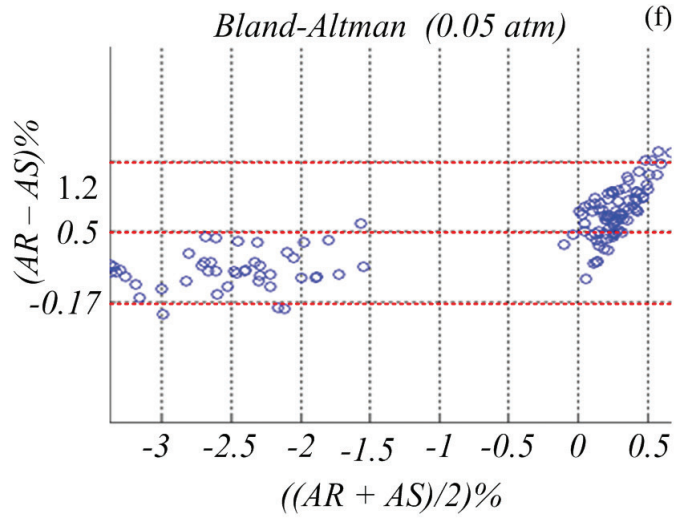

Figure 5. Bland Altman Analyses between AR and AS values for pressures of: (a) $1 \mathrm{~atm}$; (b) $0.75 \mathrm{~atm}$; (c) $0.5 \mathrm{~atm}$; (d) $0.25 \mathrm{~atm}$; (e) $0.1 \mathrm{~atm}$; (f) $0.05 \mathrm{~atm}$.

\section{Discussion}

Previous works have demonstrated the importance, and contributed to Elastography, and coronary disease investigations (Baldewsing et al., 2004; De Korte and Van der Steen, 2002; Le Floc'h et al., 2009; Liang et al., 2009; Loree et al., 1994; Maurice et al., 2005; Ophir et al., 1991). Consequently, information of Elastography, coronary and atherosclerosis, has been provided. This knowledge is important to help cardiologists and investigators to improve diagnostic, therapy, evaluation, as well as for creating new tools and methods. However, despite efforts, and advancements, atherosclerosis is still a dramatic problem. In addition, for the majority of cardiologic centers in the world, implementing, and managing the methods presented by the literature is difficult. The reason is that most of clinics and hospitals around the world lack financial support, and specialists with expertise in computing and mathematical skills. As 
Histogram (1 atm)

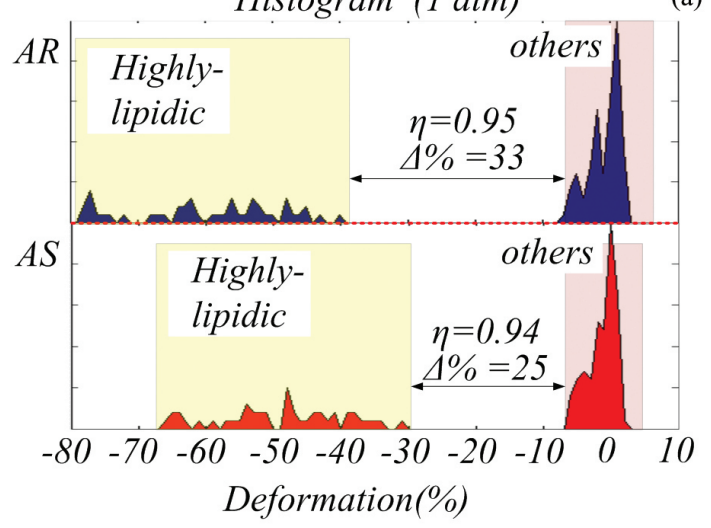

Histogram (0.5 atm)

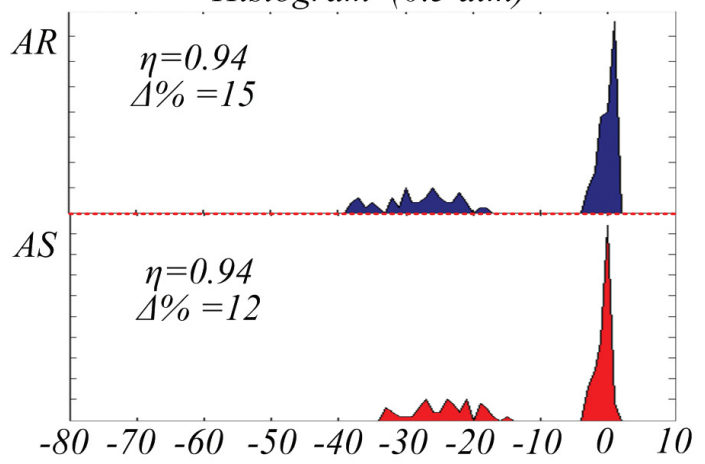

Deformation(\%)

Histogram (0.1 atm)

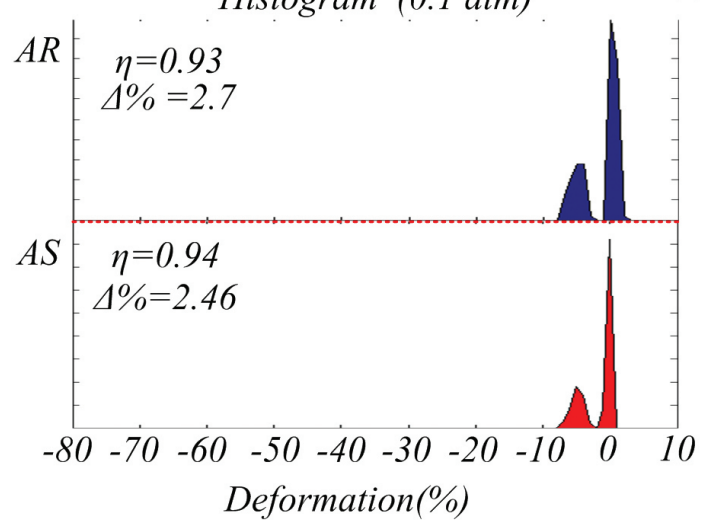

(a)

(c)

(e)
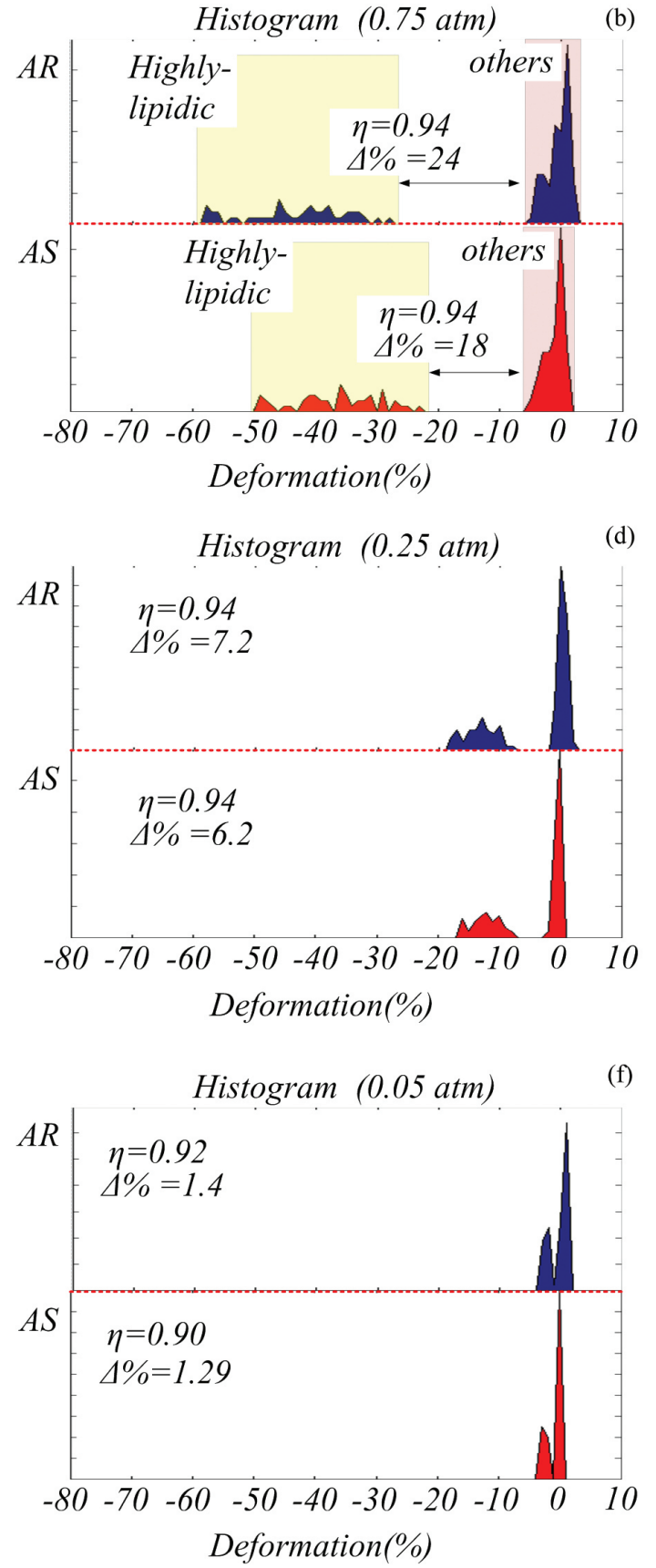

Figure 6. Histogram of the AR and AS values for pressures of: (a) $1 \mathrm{~atm}$; (b) $0.75 \mathrm{~atm}$; (c) $0.5 \mathrm{~atm}$; (d) $0.25 \mathrm{~atm}$; (e) $0.1 \mathrm{~atm}$; (f) $0.05 \mathrm{~atm}$.

a result, buying new equipment, or implementing complex methods, is not always feasible. Therefore, new approaches with simpler implementation, more practical, yet as reliable as previous methods, are welcome.

We presented a simple and practical method, in which by the area ratio of a plaque the predominant tissue is classified. The evaluation has shown that the proposed index reliably distinguishes the highly- lipidic plaque from others tissues in many situation. The methodology is based on three simple steps, Imaging Acquisition, Plaque Area Computation, and Ratio of the Plaque Area Variation. The reliability of the method was demonstrated by showing the equivalence between tissue and deformed values (Table 2), and the high correlation and agreement between deformed values computed by $A R$ and corresponding $A S$ (Figures 4 and 5). As can be seen 


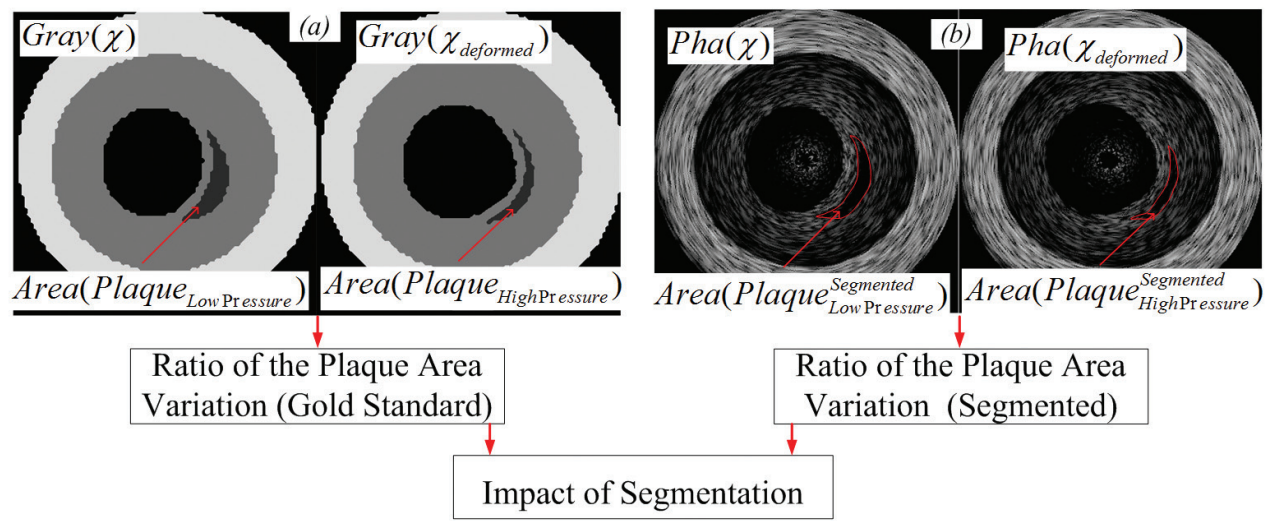

Figure 7. Computation of the impact of segmentation. (a) AR deformation computed using the gray level images, gold standards. (b) AR deformation computed using the corresponding segmented phantoms.

in Figure 4, for all plaques, and considering all situations, the $A R$ deformation values are proportional to the tissue stiffness, following the $A S$ outcomes; as a result, a correlation close to $100 \%$ was obtained. The strong agreement between the two indices is reinforced by the Bland Altman analyses, in which the great majority of points are inside the limits of agreement (Figure 5). In addition, the $A R$ distributions provide good tissue discrimination (Figure 6). The discrimination capability was quantified by computing the histogram class separability (Otsu, 1979), and the difference in distance between classes in which, the strain values computed by $A R$ provided a higher difference (Figure 6). Besides providing better discrimination among tissues, the $A R$ estimation requests a very simple area computing operation after any segmentation method. The segmentation method, for the specified acoustic parameters, provided an accuracy of $4.3 \pm 3.3 \%$, this permits reliability for all results above $0.25 \mathrm{~atm}$ of pressure, since they provide difference above $7.2 \%$. Moreover, the image processing operations can be entirely performed using basic operations of free license software, such as ImageJ.

In summary, the resultant high correlation between the proposed and well-known method, alongside the simplicity, proved the method feasibility, and low-cost. As a result, the contribution and implications are: (a) A combination of simple and usual procedures for plaque stiffness estimation; (b) The proposed method can be applied directly to similar modalities, for instance, Intravascular Optical Coherence Tomography (IOCT) and Intravascular Magnetic Resonance (IMR); (c) The proposed method may be an alternative in many places, by allowing clinics and centers to have an extra tool to support their exams, therapy planning, and evaluation.
The inclination and eccentricity of the catheter during image acquisition, not enough tissue contrast for segmentation process, may be seen as limitation for this approach. Consequently, they are also a restriction for any $2 \mathrm{D}$ algorithm, such as strain or elastic map, as they also rely only on 2D IVUS images. The Poisson's ratio, $v=0.49$, does not varies in this study, the value were obtained and used in other related studies, which considered the coronary a quasiincompressible material. Indeed, its variation would result in different deformation values, which would not represent the coronary strains, yet as this parameter is applied in the entire model and not locally these changes would be correspondent in the two indexes $A R$ and $A S$. Moreover, 3D methods would be the best choice to provide more complete vessel and coronary information, since they provide deformations of all directions, and not only the transversal one. However, 2D approaches resultant of well acquired IVUS images, with transducer carefully placed perpendicular to vessel wall, provide good information to localize and estimate predominant tissues of suspect areas. Indeed, depending on the segmentation method accuracy, higher pressure by the balloon is required, so that the separation between highly-lipidic and other tissues is increased. Therefore, the possession and correct use of a compliance balloon is an important part of the presented procedure. As a result, a simpler and alternative method to estimate mechanical properties of plaques can be used and evolved.

Finally, since we do not currently possess clinical data with desirable features and pressure information, clinical data for validation was not in the scope of this paper. In addition, computational phantoms have become a very solid tool, which are flexible tool, providing numerous investigations (Culjat et al., 2010). Accordingly, numerical phantoms were the most 
advantageous and feasible choice for the methodology evaluation. In addition, transducers with different properties may change the segmentation accuracy, since the main goal herein was to verify the two indexes equivalence, a wider analysis about the impact of segmentation depending on the transducer properties was not the scope of this paper. However, investigations using images acquired from physical phantom and clinical data, with desired coronary features and controlled parameters, will be designed and carried out with a cardiac center, and results presented in future works.

\section{Acknowledgements}

São Paulo Research Foundation - Brazil (FAPESP): Brazilian National Council of Scientific and Technological Development, (CNPq), Biomedical Engineering Laboratory of the University of São Paulo, Brazil (LEB-USP). Institute of Science and Technology of the Federal University of São Paulo, Brazil (ICT-UNIFESP).

\section{References}

Baldewsing RA, De Korte CL, Schaar JA, Mastik F, Van der Steen AFW. A finite element model for performing intravascular ultrasound Elastography of human atherosclerotic coronary arteries. Ultrasound in Medicine \& Biology. 2004; 30(6):803-13. http://dx.doi.org/10.1016/j. ultrasmedbio.2004.04.005

Cardenas DAC, Moraes MC, Furuie SS. Segmentação do lúmen em imagens de IOCT usando Fuzzy Connectedness e Reconstrução Binária Morfológica. Revista Brasileira de Engenharia Biomédica. 2013; 29(1):32-44. http://dx.doi. org/10.4322/rbeb.2013.004

Cardoso FM, Moraes MC, Furuie SS. Realistic IVUS image generation in Different Intraluminal Pressures. Ultrasound in Medicine \& Biology. 2012; 38(12):2104-19. http://dx.doi. org/10.1016/j.ultrasmedbio.2012.08.005

Céspedes EI, Ophir J, Ponnekanti H, Maklad N. Elastography: Elasticity imaging using ultrasound with application to muscle and breast in vivo. Ultrasonic Imaging. 1993; 15(2):73-88.

Choi CD, Skovoroda AR, Emelianov SY, O’Donnell M . An integrated compliant balloon ultrasound catheter for intravascular strain imaging. IEEE Transaction on Ultrasonics Ferroelectric and Frequency Control. 2002; 49(11):1552-60. http://dx.doi.org/10.1109/TUFFC.2002.1049737

Culjat MO, Goldenberg D, Tewari P, Singh RS. A review of tissue substitutes for ultrasound imaging. Ultrasound in Medicine \& Biology. 2010; 36(6):861-73. http://dx.doi. org/10.1016/j.ultrasmedbio.2010.02.012

Davies MJ. The pathophysiology of acute coronary syndromes. Heart and Education in Heart. 2000; 83(3):3616. http://dx.doi.org/10.1136/heart.83.3.361
Davies MJ. Stability and instability: two faces of coronary atherosclerosis. The Paul Dudley White lecture 1995. Circulation. 1996; 94(8):2013-20. http://dx.doi. org/10.1161/01.CIR.94.8.2013

De Korte CL, Van der Steen AFW. Intravascular ultrasound Elastography: an overview. Ultrasonics. 2002; 40:859-65. http://dx.doi.org/10.1016/S0041-624X(02)00227-5

De Korte CL, Céspedes EI, Van der Steen AFW. Influence of catheter position on estimated strain in intravascular Elastography. IEEE Transaction on Ultrasonics, Ferroelectrics, and Frequency Control. 1999; 46(3):616-25. http://dx.doi. org/10.1109/58.764848

Falk E, Shah PK, Fuster V. Coronary plaque disruption. Circulation. 1995; 92:657-71. http://dx.doi.org/10.1161/01. CIR.92.3.657

Fisher A, Gustein DE, Fayad ZA, Fuster V. Predicting plaque rupture: enhancing diagnosis and clinical decision-making in coronary artery disease. Vascular Medicine. 2000; 5:163-72.

Hoskins PR. Simulation and validation of arterial ultrasound imaging and blood flow. Ultrasound in Medicine \& Biology. 2008; 34(5):693-17. http://dx.doi.org/10.1016/j. ultrasmedbio.2007.10.017

Le Floc'h S, Ohayon J, Tracqui P, Finet G, Gharib AM, Maurice RL, Cloutier G, Pettigrew RI. Vulnerable atherosclerotic plaque elasticity reconstruction based on a segmentation-driven optimization procedure using strain measurements: Theoretical framework. IEEE Transaction on Medical Imaging. 2009; 28(7):1126-37. http://dx.doi. org/10.1109/TMI.2009.2012852

Liang Y, Zhu H, Friedman MH. The correspondence between coronary arterial wall strain and histology in a porcine model of atherosclerosis. Physics in Medicine and Biology. 2009; 54(18):5625-41. http://dx.doi. org/10.1088/0031-9155/54/18/018

Loree HM, Tobias BJ, Gibson LJ, Kamm RD, Small DM, Lee RT. Mechanical properties of model atherosclerotic lesion lipid pools. Arteriosclerosis, Thrombosis, and Vascular Biology. 1994; 14(2):230-4. http://dx.doi.org/10.1161/01. ATV.14.2.230

Maurice RL, Brusseau E, Finet G, Cloutuer G. On the potential of the Lagrangian speckle model estimator to characterize atherosclerotic plaques in endovascular Elastography: in vitro experiments using an excised human carotid artery. Ultrasound in Medicine \& Biology. 2005; 31(1):85-91. http:// dx.doi.org/10.1016/j.ultrasmedbio.2004.07.009

Moraes MC, Furuie SS. An approach to automatically segment the media-adventitia borders in IVUS. Revista Brasileira de Engenharia Biomédica. 2010; 26(3):219-33.

Moraes MC, Furuie SS. Automatic coronary wall segmentation in intravascular ultrasound images using binary morphological reconstruction. Ultrasound in Medicine \& Biology. 2011; 37(9):1486-99. http://dx.doi.org/10.1016/j. ultrasmedbio.2011.05.018

Ohayon J, Teppaz P, Finet G, Rioufol G. In-vivo prediction of human coronary plaque rupture location using intravascular ultrasound and the finite element method. 
Coronary Artery Disease. 2001; 12:655-63. http://dx.doi org/10.1097/00019501-200112000-00009

Ophir J, Céspedes EI, Ponnekanti H, Yazdi Y, Li X. Elastography. A quantitative method for imaging the elasticity of biological tissues. Ultrasonic Imaging. 1991; 13(2):111-34.

Otsu N. A threshold selection method from gray-level histograms. IEEE Transaction on Systems, Man, and Cybernetics - Part C Applications and Reviews. 1979; 9(1):626. http://dx.doi.org/10.1109/TSMC.1979.4310076

Roger VL, Go AS, Lloyd-Jones DM, Benjamin EJ, Berry JD, Borden WB, Bravata Dawn M, Dai S, Ford ES, Fox CS, Fullerton HJ, Gillespie C, Hailpern SM, Heit JA, Howard VJ, Kissela BM, Kittner SJ, Lackland DT, Lichtman JH, Lisabeth LD, Makuc DM, Marcus GM, Marelli A, Matchar DB, Moy CS, Mozaffarian D, Mussolino ME, Nichol G, Paynter NP, Soliman EZ, Sorlie PD, Sotoodehnia N, Turan TN, Virani SS, Wong ND, Woo D, Turner MB. Executive summary: heart disease and stroke statistics - 2012 update: a report from the American Heart Association. http://dx.doi.org/10.1161/ CIR.0b013e3182456d46irculation. 2012; 125(1):188-97.

Rosamond W, Flegal K, Friday G, Furie K, Go A, Greenlund K, Haase N, Ho M, Howard V, Kissela B, Kittner S, LloydJones D, McDer-mott M, Meigs J, Moy C, Nichol G, O’Donnell CJ, Roger V, Rumsfeld J, Sorlie P, Steinberger J,
Thom T, Wasserthiel-Smoller S, Hong Y. Heart disease and stroke statistics - 2007 update: a report from the American Heart Association Statistics Committee and Stroke Statistics Subcommittee. Circulation. 2007; 115(5):e69-171. http:// dx.doi.org/10.1161/CIRCULATIONAHA.106.179918

Shapo BM, Crowe JR, Skovoroda AR, Eberle MJ, Cohn NA, O'Donnell M. Displacement and strain imaging of coronary arteries with intraluminal ultrasound. IEEE Transaction on Ultrasonics, Ferroelectrics, and Frequency Control. 1996; 43(2):234-46. http://dx.doi. org/10.1109/58.485949

Viermani R, Kolodgie FD, Burke AP, Farb A, Schwartz SM. Lessons from sudden coronary death: A comprehensive morphological classification scheme for atherosclerotic lesions. Arteriosclerosis, Thrombosis, and Vascular Biology. 2000; 20:1262-75. http://dx.doi.org/10.1161/01. ATV.20.5.1262

Zienkiewicz OC, Taylor RL, Zhu JZ. The finite element method: its basis \& fundamentals. $6^{\text {th }}$ ed. Great Britain: Elsevier; 2010a.

Zienkiewicz OC, Taylor RL, Zhu JZ. The finite element method: for solid and structural mechanics. $6^{\text {th }}$ ed. Great Britain: Elsevier; 2010b.

\section{Authors}

\section{Matheus Cardoso Moraes*}

Departamento de Ciência e Tecnologia, Instituto de Ciência e Tecnologia-ICT,

Universidade Federal de São Paulo - UNIFESP, Rua Talim, 330, CEP 12231-280, São José dos Campos, SP, Brasil.

\section{Fernando Mitsuyama Cardoso, Sérgio Shiguemi Furuie}

Laboratório de Engenharia Biomédica, Departamento de Engenharia de Telecomunicações e Controle,

Escola Politécnica - Poli, Universidade de São Paulo - USP, São Paulo, SP, Brasil. 